\title{
Novel insights into the effect of paroxetine administration in pilocarpine-induced chronic epileptic rats
}

\author{
WAN-HUI LIN ${ }^{1,2^{*}}$, XIAO-FENG LI ${ }^{3 *}$, MING-XING LIN ${ }^{4 *}$, YING ZHOU $^{5}$ and HUA-PIN HUANG ${ }^{1,2}$ \\ ${ }^{1}$ Department of Neurology and Geriatrics, Union Hospital of Fujian Medical University; ${ }^{2}$ Fujian Key Laboratory of \\ Molecular Neurology, Fujian Medical University; Departments of ${ }^{3}$ Neurology and ${ }^{4}$ Pediatrics, Union Hospital of \\ Fujian Medical University; ${ }^{5}$ Neuroscience Research Center of Fujian Medical University, Fuzhou, Fujian 350001, P.R. China
}

Received July 12, 2016; Accepted May 9, 2017

DOI: $10.3892 / \mathrm{mmr} .2017 .7659$

\begin{abstract}
The aim of the present study was to investigate the role of paroxetine intervention in epilepsy, and its association with the expression of serotonin transporter (SERT) and hippocampal apoptosis. Thirty adult male Sprague Dawley rats were divided into control vehicle $(n=6)$ and epileptic $(n=24)$ groups. Status epilepticus (SE) was induced via systemic injection of pilocarpine, and seizure activity was monitored via video electroencephalogram. The epileptic group was then randomly divided into two groups; Four weeks following $\mathrm{SE}$ induction, paroxetine $(5 \mathrm{mg} / \mathrm{kg} / \mathrm{day}$; $\mathrm{SE}+$ paroxetine group) or normal saline (SE group) was intraperitoneally injected for 4 weeks. Brain tissue was collected to evaluate apoptosis via terminal deoxynucleotidyl transferase dUTP nick-end labeling. SERT, B-cell lymphoma-2 (Bcl-2) and brain derived neurotropic factor (BDNF) expression levels were evaluated by western blotting, and miR-16 expression was evaluated by reverse transcription-quantitative polymerase chain reaction. Paroxetine did not affect the mortality of the pilocarpine-induced chronic epileptic rats. Spontaneous recurrent seizures (SSRs) were observed 7-28 days following SE induction. The frequency and stage of the SSRs were reduced by paroxetine administration. Apoptotic cells were observed in the epileptic hippocampus. Following paroxetine intervention, the staining intensity and number of apoptotic cells were significantly decreased. Expression levels of BDNF and Bcl-2 were lower in the SE group compared with the vehicle group. The former was
\end{abstract}

Correspondence to: Dr Wan-Hui Lin or Dr Hua-Pin Huang, Department of Neurology and Geriatrics, Union Hospital of Fujian Medical University, 29 Xin-Quan Road, Fuzhou, Fujian 350001, P.R. China

E-mail: 21679761@qq.com

E-mail: hh-p@163.com

${ }^{*}$ Contributed equally

Key words: chronic epilepsy, miR-16, serotonin transporter, apoptosis, B-cell lymphoma-2, brain-derived neurotropic factor not altered by paroxetine injection; however, the latter was increased. In the SE group, SERT expression was not altered in the raphe nucleus but was decreased in the hippocampus. Following paroxetine administration, SERT expression was decreased in the raphe nucleus and increased in the hippocampus. In the SE group, miR-16 expression was decreased in the raphe nucleus and increased in the hippocampus. Following paroxetine administration, miR-16 expression was not altered in the raphe nucleus but was reduced in the hippocampus. In conclusion, the seizures and hippocampal apoptosis observed in chronic epileptic rats were alleviated by paroxetine treatment. This effect may be associated with the reduced Bcl-2 and BDNF expression and the modulation of SERT expression. The alterations in miR-16 expression may provide a potential explanation for the modulation of apoptosis; however, further research is required to determine the complete underlying molecular mechanism.

\section{Introduction}

Selective serotonin reuptake inhibitors (SSRIs) were previously considered to increase the occurrence of seizures (1). Previously, clinical and experimental results indicated that SSRIs alleviate the susceptibility to seizures (2-5). This effect is attributed to elevated levels of extracellular serotonin. However, the underlying molecular mechanism of this increase remains unclear. Our previous study demonstrated a downregulation of hippocampal extracellular serotonin levels in epileptic rats and impaired serotonergic neuronal function in raphe nucleus (6). The membrane bound serotonin transporter (SERT) serves an important role in modulating the metabolism of 5-hydroxytryptamine (5-HT). SSRIs target SERT in the raphe nucleus, decreasing serotonin reuptake and increasing the synaptic availability of serotonin. Therefore, the present study hypothesized that abnormal SERT expression may be present in epileptic models.

Paroxetine has been demonstrated to regulate the expression of B-cell lymphoma-2 (Bcl-2) and brain derived neurotropic factor (BDNF), which are associated with cell apoptosis and proliferation (7). In addition, mesial temporal lobe epilepsy (MTLE), the most common form of refractory epilepsy, is characterized by hippocampal sclerosis, including cell apoptosis and glial proliferation. Therefore, the present 
study hypothesized that paroxetine alleviates seizures by regulating both $\mathrm{Bcl}-2 / \mathrm{BDNF}$ and SERT.

The mechanism in which paroxetine may regulate SERT, Bcl-2 and BDNF in epilepsy remains to be fully elucidated. In recent years, an increasing number of studies, including clinical and animal experiments, demonstrated that microRNAs (miRNAs) serve an important role in the pathophysiology of epilepsy (8-11). Epileptic models are generally accompanied by selective alterations in miRNAs that regulate neuronal death, ion channels and inflammation (12-15). In a genome wide miRNA profiling study, microRNA (miR)-16 was increased in hippocampal tissue collected from patients with MTLE (15). One study revealed that paroxetine upregulates miR-16 expression in the raphe nucleus (7), and another demonstrated that Bcl-2 expression was negatively associated with miR-16 expression (16). These results were obtained using animal models of depression or tumor cells.

The present study focused on pilocarpine-induced chronic epileptic rats. Firstly, the effects of paroxetine on spontaneous recurrent seizures (SSRs) and hippocampal apoptosis was investigated. Secondly, SERT, Bcl-2 and BDNF expression levels were evaluated using western blotting, and miR-16 expression was evaluated using reverse transcription-quantitative polymerase chain reaction. Finally, the underlying molecular mechanism of miR-16 in the pathogenesis of epilepsy was investigated.

\section{Materials and methods}

Pilocarpine model of chronic epilepsy. The present study was performed in accordance with the Guide for the Care and Use of Animal Experimentation of Fujian Medical University and the Fujian Medical University Animal Ethics Committee (Fuzhou, China) specifically approved this study. Surgery was performed using $10 \%$ chloral hydrate anesthesia and efforts were made to minimize suffering. Male adult (8-10 weeks) Sprague Dawley rats (220-250 g) were housed under standard conditions (temperature, $22-26^{\circ} \mathrm{C}$; 12-h light/dark cycle; humidity, 45-50\%) and had free access to food and water. Thirty rats were divided into two groups (6 rats in the vehicle group and 24 rats in the epileptic group). One week prior to the induction of status epilepticus (SE), surface electrodes were implanted into the skulls of the rats under $10 \%$ chloral hydrate anesthesia as previously described (6). A frontal electrode was implanted above the frontal cortex [coordinates, $2.5 \mathrm{~mm}$ frontal; $2.0 \mathrm{~mm}$ left and $0.5 \mathrm{~mm}$ deep from the bregma (17)], a second electrode was fixed to the surface of the skull as a ground electrode and a third electrode was fixed behind the ear as a reference electrode. Following the implantation procedure, animals were intraperitoneally (i.p.) injected with gentamicin to prevent infection and were allowed to recover from surgery for 1 week prior to experimentation. Twenty minutes prior to injection of pilocarpine, the muscarinic antagonist, atropine, was administered i.p. (1 mg/kg) to reduce the adverse peripheral effects of pilocarpine. The rats were injected i.p. with pilocarpine $(30 \mathrm{mg} / \mathrm{kg}$; Sigma-Aldrich; Merck KGaA, Darmstadt, Germany) 16-18 $\mathrm{h}$ after the administration of lithium $(127 \mathrm{mg} / \mathrm{kg}$, i.p.). After the drugs were administered, the progressive evolution of seizure behavior was observed and rated according to the Racine scale (18). The Racine scale was used to rate the stage of epilepsy: Stage 1 was characterized by behavioral arrest; stage 2 by head nodding, gnawing, and mild tremors; stage 3 by unilateral forelimb clonus; stage 4 by bilateral forelimb clonus; and stage 5 by severe seizures with prolonged loss of postural control or prolonged clonus. Only animals that developed stage IV and V seizures were used. SE was defined as the persistence of stage IV and V seizures for longer than $30 \mathrm{~min}$ (18). Electroencephalogram (EEG) potentials and the behavior of the animals were monitored using a video monitoring system (Biopac Systems Inc., Goleta, CA, USA) three times a day for $2 \mathrm{~h}$ each session for 8 weeks after the establishment of SE. During the chronic period, the SSRs were evaluated based on frequency (times/week) and stage (19). EEG discharges with amplitudes exceeding $50 \mu \mathrm{V}$, which was typically twice the basal EEG discharge amplitude, and spikes ( $\leq 70 \mathrm{msec})$ and sharp waves (70-200 msec) were counted as seizure discharges.

Intervention. A total of 18 rats survived $\mathrm{SE}$ induction however, four weeks after the induction of SE only 14 rats had survived. These 14 epileptic rats were divided into two sub-groups: SE and SE + paroxetine. The former group received normal saline (NS) as a control, and the latter received paroxetine. Paroxetine ( $5 \mathrm{mg} / \mathrm{kg} /$ day) or NS was injected i.p. for 4 weeks; only 12 rats survived during the final experiment.

Brain region isolation and morphological examinations. At the end of the experiments, the rats were deeply anesthetized (10\% chloral hydrate, $2 \mathrm{ml} / \mathrm{kg}$ ) and transcardially perfused with $0.1 \mathrm{mmol} / \mathrm{l} \mathrm{PBS}$ ( $\mathrm{pH} 7.4$ ). If the tissue required fixation, the rats were perfused with PBS followed by $4 \%$ paraformaldehyde. One portion of the hippocampal tissue was used for terminal deoxynucleotidyl transferase dUTP nick-end labelling TUNEL/horseradish peroxidase (HRP) staining to visualize apoptotic cells. Another portion of the hippocampal tissue and a sample of the raphe nucleus tissue were used to evaluate expressions of SERT, Bcl-2 and BDNF via western blotting. A second set of hippocampal/raphe nucleus tissue samples was used to analyze expression of miR-16 via reverse transcription-quantitative polymerase chain reaction (RT-qPCR).

Immunohistochemical staining. The unilateral hippocampal tissue from the six rats in each of the control, SE and $\mathrm{SE}+$ paroxetine groups was rapidly isolated, fixed with $4 \%$ paraformaldehyde for $48 \mathrm{~h}$ at $4^{\circ} \mathrm{C}$ and embedded in paraffin. Next, the paraffin-embedded tissue was cut into sections including the cornu ammonis (CA) 1-3 and the dentate gyrus (DG) regions of the hippocampus. Subsequently, the slices were dewaxed in a series of alcohols and incubated with proteinase $\mathrm{K}$ $(20 \mu \mathrm{g} / \mathrm{ml})$ for $10 \mathrm{~min}$ at room temperature $\left(22-28^{\circ} \mathrm{C}\right)$, terminal deoxynucleotidyl transferase $(50 \mu \mathrm{l})$ at $37^{\circ} \mathrm{C}$ for $60 \mathrm{~min}$ and an anti-biotin HRP solution at $37^{\circ} \mathrm{C}$ for $30 \mathrm{~min}$. Finally, diaminobenzidine was used for color development and hematoxylin was used for counterstaining at $37^{\circ} \mathrm{C}$ for $15 \mathrm{~min}$. The stained brain sections were observed using a Leica DM2500 microscope (Leica Microsystems GmbH, Wetzlar, Germany), and images were captured using a digital camera and Leica software version 3.7 (Leica Microsystems GmbH). For quantification, five fields of view at $\mathrm{x} 400$ magnification were 
randomly examined, and the number of brown cells in each field was counted by independent blinded operators. Brown spots were counted irrespective of whether they contained a blue nucleus. An immunohistochemical score (IHS) was calculated by multiplying the number of immunoreactive cells (quantity score) by the staining intensity (staining intensity score). Quantity scores were estimated as follows: No staining, $0 ; 1-10 \%$ of cells, $1 ; 11-50 \%, 2 ; 51-80 \%, 3 ; 81-100 \%$, 4. Staining intensity was rated on a scale of $0-3$ where: 0 , negative; 1 , weak; 2 , moderate; and 3, strong. The IHS ranged from 0 to 12 .

Western blotting. Proteins were extracted from the hippocampal and raphe nucleus tissue using cytoplasmic extracts (Beyotime Institute of Biotechnology, Jiangsu, China) and 10X PMSF (100:1). Protein concentration was detected using a bicinchoninic acid working solution (Beyotime Institute of Biotechnology) according to the manufacturer's instructions. A total of $30 \mu \mathrm{g}$ protein/lane was separated via 10\% SDS-PAGE and transferred onto a nitrocellulose membrane. After blocking of the membranes with $5 \%$ skim milk powder for $2 \mathrm{~h}$ at room temperature, they were incubated at $4^{\circ} \mathrm{C}$ overnight in primary antibodies against the following target proteins: SERT (cat. no. ab172884, 1:1,000, polyclonal rabbit; Abcam, Cambridge, UK), Bcl-2 (cat. no. AB112-1, 1:1,000, monoclonal rabbit; Beyotime Institute of Biotechnology), BDNF $(1: 1,000$, polyclonal rabbit, cat. no. ab75040; Abcam, Cambridge, MA, USA), and $\beta$-actin (cat. no. EM32011-02, 1:1,000, monoclonal mouse; Beijing Emarbio Science and Technology, Beijing, China; www.emarbio.com). Subsequently, the membranes were washed and incubated in species-specific peroxidase-conjugated secondary antibodies for $2 \mathrm{~h}$ at room temperature. The secondary antibodies (all 1:6,000; HRP-conjugated) used to distinguish SERT, Bcl-2, BDNF (anti-rabbit, cat. no. A0208; Beyotime Institute of Biotechnology) and $\beta$-actin (anti-mouse, cat. no. A0216; Beyotime Institute of Biotechnology) were all produced in goats. The specific bands were detected using an Enhanced Chemiluminescence system (GE Healthcare, Chicago, IL, USA) and a Bio-Rad electrophoresis image analyzer (Bio-Rad Laboratories, Inc., Hercules, CA, USA) and analyzed using ImageJ software (National Institutes of Health, Bethesda, MD, USA)

$R T-q P C R$. Total RNA was extracted from hippocampal and raphe nucleus tissue samples using TRIzol ${ }^{\circledR}$ Reagent (cat. no. 15596-026; Invitrogen; Thermo Fisher Scientific, Inc., Waltham, MA, USA) according to the manufacturer's instructions and quantified using a spectrophotometer (NanoDrop2000/2000C; Thermo Fisher Scientific, Inc.). Subsequently, the RNA was reverse transcribed into cDNA using M-MLV Reverse Transcriptase (cat. no. M1705; Promega Corporation, Madison, WI, USA) according to the manufacturer's instructions and amplified using a Real-Time PCR Mx3000p Instrument (Agilent Technologies, Inc., Santa Clara, CA, USA). RT-qPCR was performed using SYBR ${ }^{\circledR}$ Premix Ex Taq ${ }^{\mathrm{TM}}$ (with a pre-denaturation at $95^{\circ} \mathrm{C}$ for $30 \mathrm{sec}$, followed by 40 cycles of denaturation at $95^{\circ} \mathrm{C}$ for $5 \mathrm{sec}$, primer annealing at $60^{\circ} \mathrm{C}$ for $30 \mathrm{sec}$; acquisition of the dissolve curve at $95^{\circ} \mathrm{C}$ for $15 \mathrm{sec}$; at $60^{\circ} \mathrm{C}$ for $30 \mathrm{sec}$; at $95^{\circ} \mathrm{C}$ for $15 \mathrm{sec}$ ) (cat. no. DRR041B; Takara Biotechnology Co., Ltd., Dalian, China). The primers for the target gene (forward primer, cat. no. SSD809230873; downstream primer, cat. no. SSD089261711; and reverse primer, cat. no. SSD809230181) and the reference gene U6 (forward primer, cat. no. SSD0904071006; downstream primer, cat. no. SSD0904071007; and reverse primer, cat. no. SSD904071008) were designed and synthesized by Guangzhou RiboBio Co., Ltd (Guangzhou, China). After PCR, a melting curve was obtained to assess the quality of the reaction. The relative expression of miRNA was calculated as follows: $2^{-\Delta \Delta \mathrm{Cq}}(\Delta \mathrm{Ct}=\mathrm{Cq}(\mathrm{TG})-\mathrm{Cq}(\mathrm{RG}) ; \Delta \Delta \mathrm{Cq}=\Delta \mathrm{Cq}$ (experimental) - $\Delta \mathrm{Cq}$ (control) (20).

Materials. Atropine, pilocarpine hydrochloride, paroxetine, trypsin, paraformaldehyde and the monoclonal antibody for BDNF were purchased from Sigma-Aldrich; Merck KGaA. The specific antibodies for Bcl-2, SERT and $\beta$-actin were purchased from Abcam. The TUNEL/HRP kit was purchased from Roche Applied Science (Penzberg, Germany). All other reagents were purchased from Biyuantian (Jiangsu, China).

Statistical analysis. Data are presented as the mean \pm standard deviation. Statistical analysis was performed using GraphPad Prism v6.0 software (GraphPad Software, Inc., La Jolla, CA, USA). One-way analysis of variance was performed with multiple comparisons between the groups using an Dunnett's post hoc tests and comparisons between the attack levels were performed using the Mann-Whitney test method. $\mathrm{P}<0.05$ was considered to indicate a statistically significant difference.

\section{Results}

Behavioral alterations in chronic epileptic rats. At 3-10 min following the administration of pilocarpine, the animals exhibited masticatory movements, salivation, sniffing movements, tremors and partial seizures. At 15-30 min following pilocarpine injection, the animals developed SE that persisted for longer than $30 \mathrm{~min}$. SE was successfully induced in all the epileptic group rats. The acute phase was followed by a quiescent phase of 2-7 days in which the animals behaved normally except for anorexia and hypokinesis. SSR-like activity was observed 8-27 days after the induction of SE. A total of 14 rats survived the induction of SE at 4 weeks; subsequently, 7 rats received paroxetine treatment and the other 7 rats were untreated. At the end of the experiment, 6 rats survived in each of the paroxetine-treated and untreated groups (total, $n=12$ rats). The frequency per week $(\mathrm{P}<0.0001$; Fig. 1A) and the mean stage of the SSRs $(\mathrm{P}<0.05$; Fig. 1B) were significantly decreased after paroxetine intervention compared with the SE group. The mortality of the rats may have been due to pilocarpine-induced epilepsy.

TUNEL/HRP staining in the hippocampus. Tissue sections of the hippocampus from the experimental groups were stained with TUNEL/HRP in order to evaluate apoptosis. With this assay, apoptotic neurons in the DG region were positively stained (brown) in the cytoplasm. In the vehicle group, positively stained neurons were sparse, and those that were positive only exhibited light positive staining (Fig. 2A). The number and IHS score of apoptotic neurons were increased in the SE group compared with the vehicle group $(\mathrm{P}<0.0001$ and $\mathrm{P}<0.001$, respectively; Fig. $2 \mathrm{~B}$ and $\mathrm{C}$ ). Following paroxetine 
A

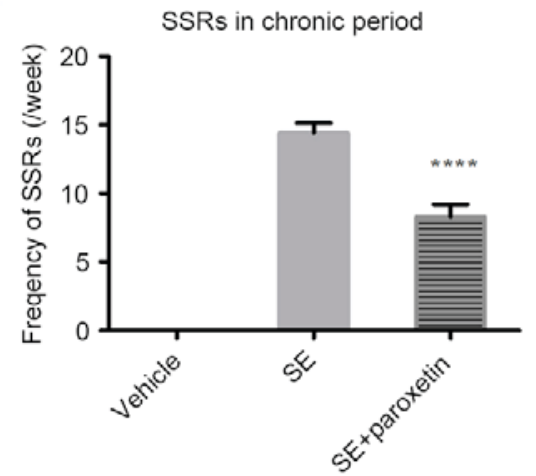

B

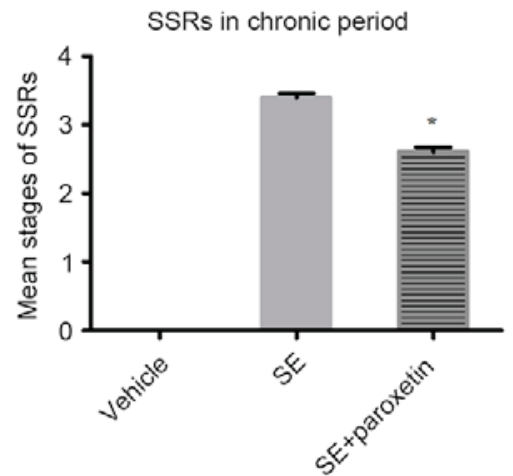

Figure 1. Behavioral alterations in chronic epileptic rats. (A) Frequency of SSRs per week in the chronic period. (B) Mean stage of SSRs in the chronic period. Data are presented as the mean \pm standard deviation. ${ }^{*} \mathrm{P}<0.05$ and ${ }^{* * * * *} \mathrm{P}<0.0001$ vs. SE. SSRs, spontaneous recurrent seizures; SE, status epilepticus.

A

Vehicle
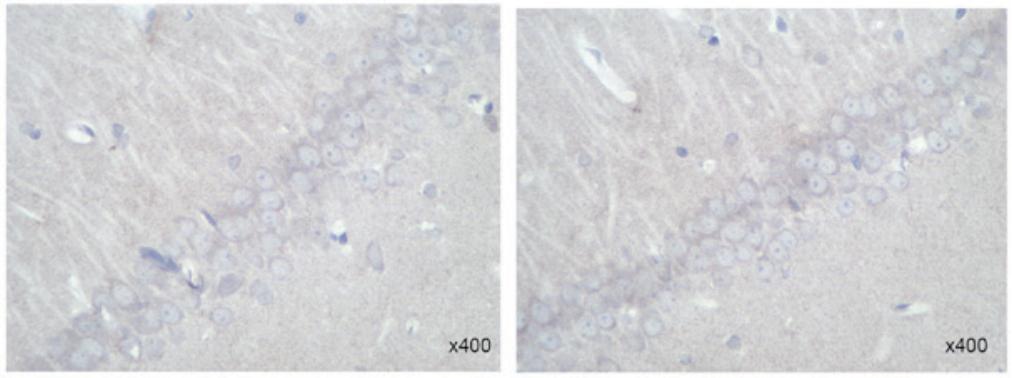

SE
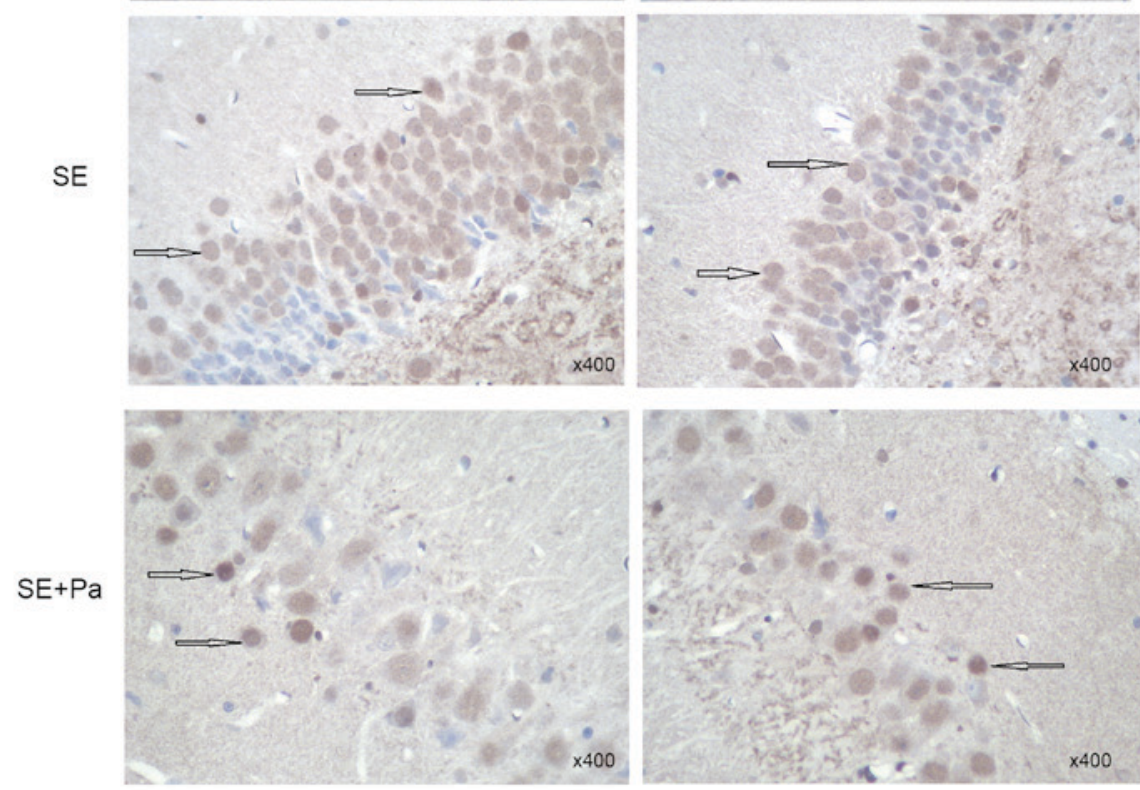

B

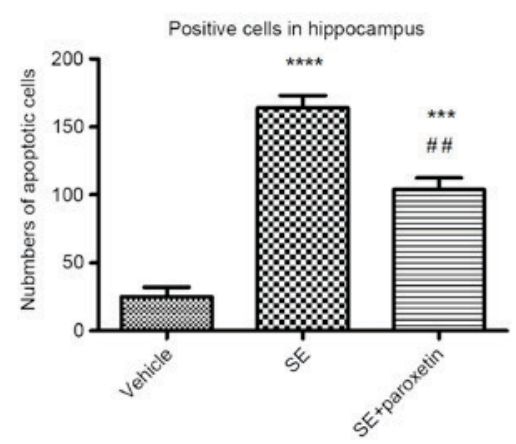

C

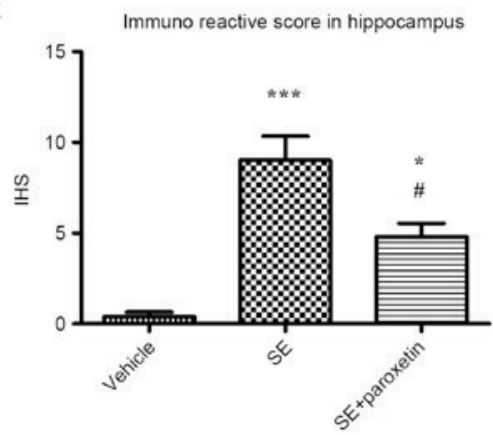

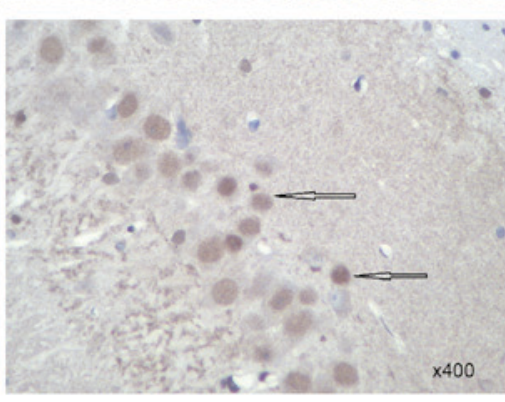

Figure 2. Terminal deoxynucleotidyl transferase dUTP nick-end labelling/horse radish peroxidase staining in the hippocampus. (A) Representative staining images. Brown cells denote the apoptotic cells (examples pointed to by arrows; magnification, x400). (B) Quantification of the number of brown cells in 5 high power fields. (C) Quantification of the IHS scoring in the experimental groups. Data are presented as the mean \pm standard deviation. ${ }^{* * *} \mathrm{P}<0.001$ and ${ }^{* * * * *} \mathrm{P}<0.0001$ vs. vehicle; ${ }^{\#} \mathrm{P}<0.05$ and ${ }^{\#} \mathrm{P}<0.01$ vs. SE. IHS, immunohistochemical score; SE, status epilepticus; Pa, paroxetine. 


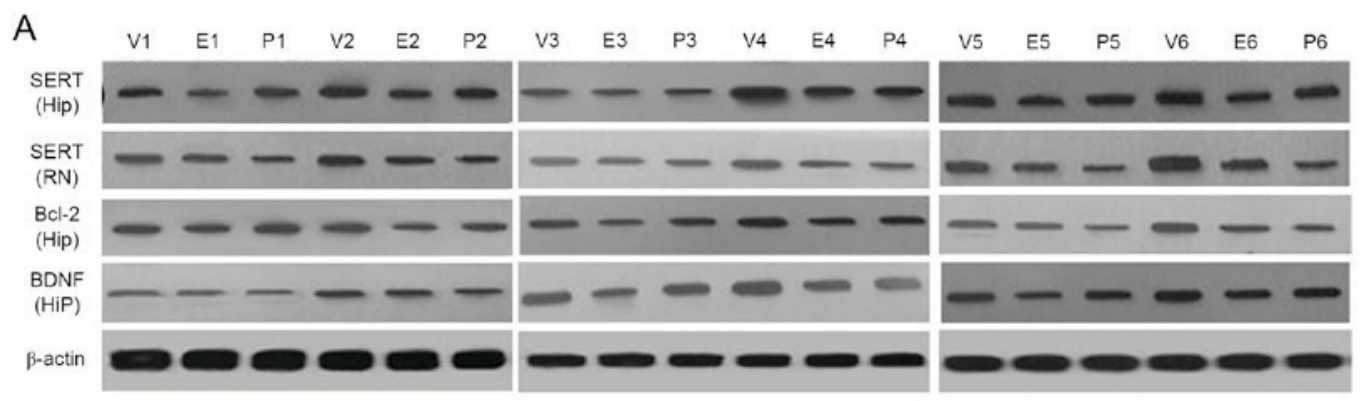

B

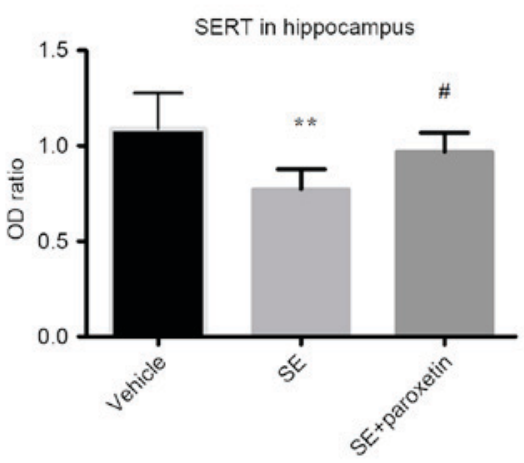

D

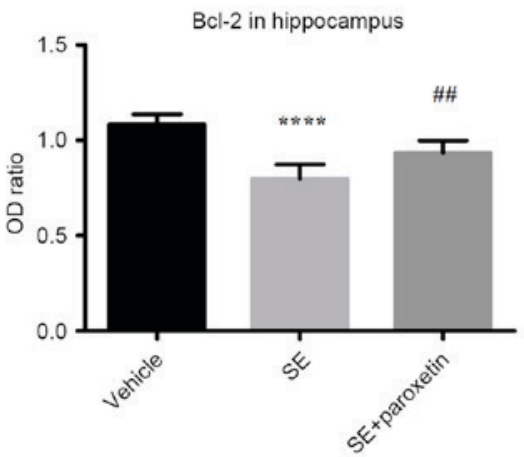

C

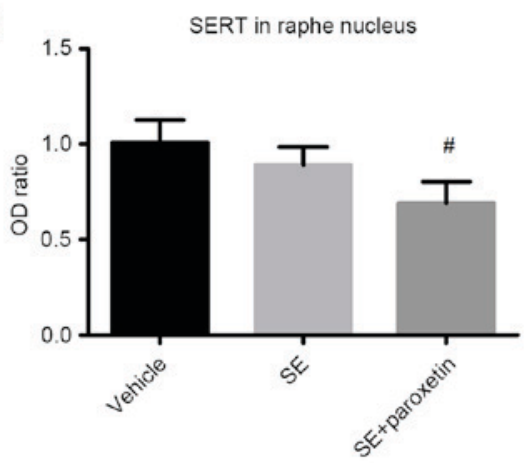

E

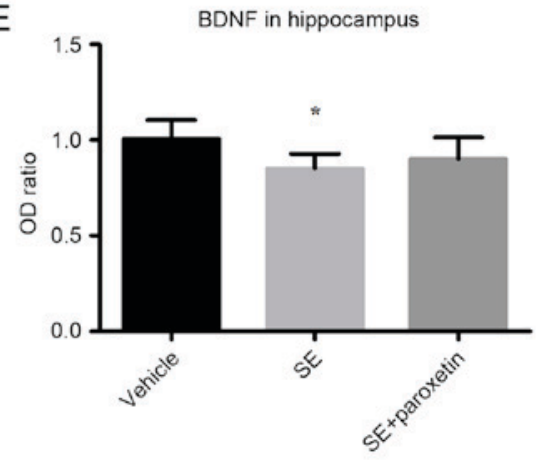

Figure 3. Protein expression levels of SERT, Bcl-2 and BDNF detected by western blotting. (A) Blot images of samples from 6 animals per experimental group (V1-6, vehicle group; E1-6, SE group; P1-6, SE + paroxetine group). Quantification of protein signals was performed for (B) SERT expression in the hippocampus, (C) SERT expression in the raphe nucleus, (D) Bcl-2 in the hippocampus and (E) BDNF expression in the hippocampus. Data are presented as the mean \pm standard deviation. ${ }^{*} \mathrm{P}<0.05,{ }^{* *} \mathrm{P}<0.01$ and ${ }^{* * * * *} \mathrm{P}<0.0001$ vs. vehicle; ${ }^{\#} \mathrm{P}<0.05$ and ${ }^{\# \#} \mathrm{P}<0.01$ vs. SE. SERT, serotonin transporter; Bcl-2, B-cell lymphoma-2; BDNF, brain derived neurotropic factor; SE, status epilepticus; Hip, hippocampus; RN, raphe nucleus.

intervention, the number of apoptotic neurons and IHS score significantly decreased compared with the SE group $(\mathrm{P}<0.001$ and $\mathrm{P}<0.05$, respectively; Fig. $2 \mathrm{~B}$ and $\mathrm{C}$ ).

Expression of SERT, Bcl-2 and BDNF proteins. SERT was expressed in both the raphe nucleus and the hippocampus in all experimental groups (Fig. 3A). In the hippocampus, SERT expression in the SE group was decreased compared with the vehicle group $(\mathrm{P}<0.01$; Fig. $3 \mathrm{~A}$ and $\mathrm{B})$, but this effect was reversed by paroxetine, with SERT expression being significantly increased in the $\mathrm{SE}+$ paroxetine group compared with the SE group $(\mathrm{P}<0.05$; Fig. 3A and $\mathrm{B})$. In the raphe nucleus, SERT expression was decreased in the $\mathrm{SE}+$ paroxetine group compared with the SE group $(\mathrm{P}<0.05$; Fig. 3A and $\mathrm{C})$. The pattern of differences in Bcl-2 expression in the hippocampus was similar to that of SERT expression. Bcl-2 expression levels in the SE group were decreased compared with the vehicle group $(\mathrm{P}<0.0001$; Fig. 3A and D), but following paroxetine intervention, Bcl-2 expression levels were significantly increased compared with the SE group $(\mathrm{P}<0.01$; Fig. $3 \mathrm{~A}$ and $\mathrm{D})$. Additionally, in the hippocampus, BDNF expression levels in the SE group were decreased compared with the vehicle group $(\mathrm{P}<0.05$; Fig. 3A and E); however, paroxetine intervention did not significantly alter BDNF expression compared with the SE group (Fig. 3A and D).

Expression of $m i R-16$. In the hippocampus, miR-16 expression in the SE group was increased compared with the vehicle group $(\mathrm{P}<0.0001$; Fig. 4A). Following paroxetine administration, miR-16 expression was significantly decreased compared with the SE group ( $\mathrm{P}<0.05$; Fig. 4A); however, miR-16 expression remained higher in the $\mathrm{SE}+$ paroxetine group than in the vehicle group $(\mathrm{P}<0.001$; Fig. 4A). In the raphe nucleus, miR-16 expression in the SE group was decreased compared with the vehicle group $(\mathrm{P}<0.0001$; Fig. 4B), and this increased expression was not significantly altered following paroxetine administration (Fig. 4B). 
A

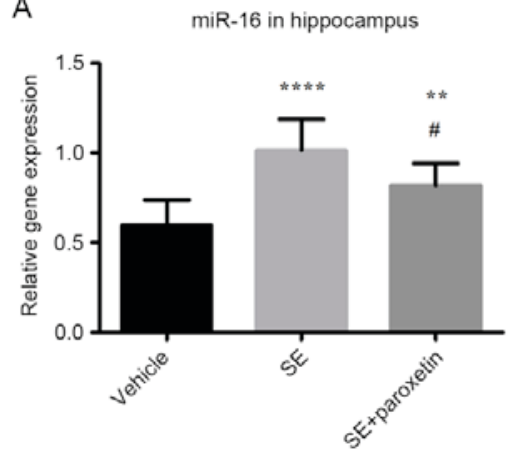

B

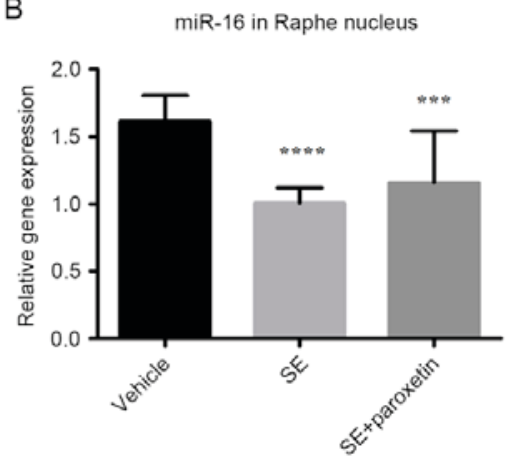

Figure 4. Expression of miR-16 detected by reverse transcription-quantitative polymerase chain reaction in (A) the hippocampus and (B) the raphe nucleus. Data are presented as the mean \pm standard deviation. ${ }^{* *} \mathrm{P}<0.01,{ }^{* * *} \mathrm{P}<0.001$ and ${ }^{* * * * *} \mathrm{P}<0.0001$ vs. vehicle; ${ }^{*} \mathrm{P}<0.05$ vs. SE. SE, status epilepticus.

\section{Discussion}

Previous studies have demonstrated that serotonin serves an important role in epilepsy (21-23). In general, drugs that increase the level of extracellular serotonin, such as SSRIs/tryptophan and 5-hydroxytryptophan (5-HT) (21,24,25), alleviate seizures, whereas drugs such as 5,7-dihydroxytryptamine that reduce the level of serotonin may aggravate seizures $(26,27)$. However, the effects of SSRIs on seizures remain controversial. Previous clinical tests suggested that long-term treatment of depression with SSRIs increases the incidence of epilepsy. The rate of epileptogenesis in rats has been demonstrated to be enhanced by chronic SSRI treatment (28). However, clinical and animal experiments have demonstrated that SSRIs may decrease seizures, and these drugs are considered safe for use in epilepsy $(3-5,25)$. In the present study, four weeks of paroxetine treatment alleviated seizures in pilocarpine-induced chronic epileptic rats. Further studies will be required to determine the longer-term effects of SSRI treatment on epilepsy.

The molecular mechanism underlying the therapeutic role of SSRIs in epilepsy remains unclear. Our previous study revealed that in pilocarpine-induced epileptic rats, the level of extracellular serotonin in the hippocampus decreased, as did the number of 5-HTP-positive neurons in the raphe nucleus (6). SERT, which modulates 5-HT metabolism, is considered important for epilepsy, especially when it is accompanied by depression (29-32). Therefore, we hypothesized that SERT is abnormally expressed in pilocarpine-induced epileptic rats, although one study demonstrated the absence of a significant change in mRNA expression levels of SERT in this model (28). The present study demonstrated that SERT is expressed not only in the raphe nucleus but also in the hippocampus. No significant decreases in SERT expression in the raphe nucleus was observed in pilocarpine-induced chronic epileptic rats. Theoretically, it should decrease due to impairment of the raphe nucleus. It was hypothesized that the absence of an alteration in SERT expression reflects a form of self-regulation to ensure the availability of serotonin. Following paroxetine intervention, SERT was downregulated in the raphe nucleus, decreasing reuptake and thus increasing synaptic 5-HT availability. Additionally, it was downregulated in the hippocampus in epileptic rats. This result is consistent with the results of Martinez et al (31), who demonstrated that SERT activity in the insula and fusiform gyrus was reduced in patients with temporal lobe epilepsy accompanied by depression. Following paroxetine intervention, SERT was upregulated in the hippocampus, indicating increased reuptake and therefore an increased level of serotonin in the hippocampus. Therefore, SERT expression alterations in pilocarpine-induced chronic epileptic rats differed across brain regions, and paroxetine treatment modulated the expression of SERT to increase the level of extracellular serotonin in the hippocampus.

The question remains as to why SERT expression levels are altered in epilepsy. Previous studies have focused on the epigenetic and genetic pathogenesis of epilepsy (33-36), under the assumption that one gene modulates a number of proteins and one protein may be regulated by various different genes. miRNA is a one example, as selective alterations in miRNAs that regulate neuronal cell death, ion channels and inflammation have been identified in epileptic patients and in experimental epileptogenic models $(8,10,11,13,37,38)$. In a genome wide miRNA profiling study, miR-16 expression was increased in the hippocampus of patients with MTLE (9). Similarly, the present study demonstrated that miR-16 was upregulated in the hippocampus in pilocarpine-induced chronic epileptic rats. However, following paroxetine intervention, it was downregulated. By contrast, in the raphe nucleus, miR-16 was downregulated, demonstrating that the alteration in miR-16 expression in chronic epileptic rats had brain tissue specificity. The pattern of change in miR-16 expression was opposite to that of SERT. In addition, miR-16 has been reported to target SERT, and in experimental models of depression, paroxetine may upregulate miR-16 expression in the raphe nucleus (7). Therefore, it may be hypothesized that miR-16 may have a role in regulating the gene expression of SERT in the raphe nucleus and hippocampus of chronic epileptic rats.

Another question that remains is whether other proteins are targeted by miR-16. Recent experimental results have suggested that miR-16 may regulate the cell cycle and apoptosis in tumors $(16,39,40)$, including $\mathrm{T}$ lymphoblastic lymphoma/leukemia, breast cancer, glioma and hepatocellular carcinoma. For example, Mobarra et al (41) revealed that miR-16 overexpression reduces Cyclin D1 and Bcl-2 expression and increases apoptosis in breast cancer cells. Recent studies have demonstrated that miR-16 may target $\operatorname{BDNF}(42,43)$. In general, miR-16 overexpression may downregulate BDNF and therefore inhibit cell proliferation, including in depression models and SH-SY5Y cells $(42,43)$. Temporal lobe epilepsy 
is characterized by hippocampal sclerosis, including neuronal apoptosis and glial proliferation. As an antiapoptotic protein, Bcl-2 has been demonstrated to regulate mitochondrial permeability and caspase- 3 activity in epilepsy $(44,45)$, whereas the association between BDNF and seizures remains controversial. One study revealed that upregulating BDNF may increase epilepsy susceptibility (46), and another reported that upregulating BDNF alleviates seizures in pilocarpine-induced epileptic mice (47). This suggested that continuously injecting an appropriate amount of BDNF into the hippocampus may alleviate kainic acid-induced seizures via the promotion of neuronal regeneration and therefore demonstrated that BDNF serves a protective role in neuronal apoptosis (47). In the present study, obvious neuronal apoptosis and downregulation of Bcl-2 and BDNF expression were observed in pilocarpine-induced chronic epileptic rats. Paroxetine alleviated neuronal apoptosis and upregulated $\mathrm{Bcl}-2$ expression. In the present study, Bcl-2 exhibited an opposite trend of expression than miR-16, and therefore it may be hypothesized that miR-16 overexpression downregulated Bcl-2 expression and increased neuronal apoptosis in chronic epileptic rats. However, the association between BDNF and miR-16 in epileptic rats remains uncertain.

The mechanism by which miR-16 targets SERT, Bcl-2 and BDNF requires further study. In addition, one protein may be regulated by a number of miRNAs. For example, SERT is regulated by miR-16, in addition to miR-55 and other miRNAs $(48,49)$. Therefore, further studies are required to determine whether SERT, Bcl-2 and BDNF are primarily targeted by miR-16.

In conclusion, the present study demonstrated that seizures and hippocampal apoptosis in chronic epileptic rats may be alleviated by paroxetine treatment, which may be associated with alterations in SERT and Bcl-2/BDNF protein expression. The alterations in miR-16 expression may provide a potential explanation for the modulation of apoptosis. Further study is required to determine the underlying molecular mechanisms.

\section{Acknowledgements}

The present study was supported by the National Natural Science Foundation of China (grant no. 81371426), the Health Department Youth Foundation of Fujian Province (grant no. 2013-1-26) and was sponsored by the Key Clinical Specialty Discipline Construction Program of Fujian, China. Thanks to Dr Edward C. Mignot (Shandong University) for linguistic advice.

\section{References}

1. Curran S: Effect of paroxetine on seizure length during electroconvulsive therapy. Acta Psychiatr Scand 92: 239-240, 1995.

2. Alper K, Schwartz KA, Kolts RL and Khan A: Seizure incidence in psychopharmacological clinical trials: An analysis of Food and Drug Administration (FDA) summary basis of approval reports. Biol Psychiatry 62: 345-354, 2007.

3. Payandemehr B, Ghasemi M and Dehpour AR: Citalopram as a good candidate for treatment of depression in patients with epilepsy. Epilepsy Behav 44: 96-97, 2015.

4. Shiha AA, de Cristóbal J, Delgado M, Fernández de la Rosa R, Bascuñana P, Pozo MA and García-García L: Subacute administration of fluoxetine prevents short-term brain hypometabolism and reduces brain damage markers induced by the lithium-pilocarpine model of epilepsy in rats. Brain Res Bull 111: 36-47, 2015 .
5. Vermoesen K, Massie A, Smolders I and Clinckers R: The antidepressants citalopram and reboxetine reduce seizure frequency in rats with chronic epilepsy. Epilepsia 53: 870-878, 2012.

6. Lin WH, Huang HP, Lin MX, Chen SG, Lv XC, Che CH and Lin JL: Seizure-induced 5-HT release and chronic impairment of serotonergic function in rats. Neurosci Lett 534: 1-6, 2013.

7. Launay JM, Mouillet-Richard S, Baudry A, Pietri M and Kellermann O: Raphe-mediated signals control the hippocampal response to SRI antidepressants via miR-16. Transl Psychiatry 1: e56, 2011.

8. Wang J, Yu JT, Tan L, Tian Y, Ma J, Tan CC, Wang HF, Liu Y, Tan MS, Jiang T and Tan L: Genome-wide circulating microRNA expression profiling indicates biomarkers for epilepsy. Sci Rep 5: 9522,2015

9. Li MM, Jiang T, Sun Z, Zhang Q, Tan CC, Yu JT and Tan L: Genome-wide microRNA expression profiles in hippocampus of rats with chronic temporal lobe epilepsy. Sci Rep 4: 4734, 2014.

10. Henshall DC: MicroRNA and epilepsy: Profiling, functions and potential clinical applications. Curr Opin Neurol 27: 199-205, 2014.

11. Hu K, Xie YY, Zhang C, Ouyang DS, Long HY, Sun DN, Long LL, Feng L, Li Y and Xiao B: MicroRNA expression profile of the hippocampus in a rat model of temporal lobe epilepsy and miR-34a-targeted neuroprotection against hippocampal neurone cell apoptosis post-status epilepticus. BMC Neurosci 13: 115, 2012.

12. Omran A, Peng J, Zhang C, Xiang QL, Xue J, Gan N, Kong H and Yin F: Interleukin-1 $\beta$ and microRNA-146a in an immature rat model and children with mesial temporal lobe epilepsy. Epilepsia 53: 1215-1224, 2012.

13. Reschke CR and Henshall DC: microRNA and epilepsy. Adv Exp Med Biol 888: 41-70, 2015

14. Ashhab MU, Omran A, Kong H, Gan N, He F, Peng J and Yin F: Expressions of tumor necrosis factor alpha and microRNA-155 in immature rat model of status epilepticus and children with mesial temporal lobe epilepsy. J Mol Neurosci 51: 950-958, 2013.

15. Kan AA, van Erp S, Derijck AA, de Wit M, Hessel EV, O'Duibhir E, de Jager W, Van Rijen PC, Gosselaar PH, de Graan PN and Pasterkamp RJ: Genome-wide microRNA profiling of human temporal lobe epilepsy identifies modulators of the immune response. Cell Mol Life Sci 69: 3127-3145, 2012.

16. Lin K, Farahani M, Yang Y, Johnson GG, Oates M, Atherton M, Douglas A, Kalakonda N and Pettitt AR: Loss of MIR15A and MIR16-1 at 13q14 is associated with increased TP53 mRNA, de-repression of BCL2 and adverse outcome in chronic lymphocytic leukaemia. Br J Haematol 167: 346-355, 2014.

17. Paxinos G and Watson C: The Rat Brain in Stereotactic Coordinates. 5th edition. Elsevier Academic Press, Boston, MA, pp161, 2005.

18. Racine RJ: Modification of seizure activity by electrical stimulation. II. Motor seizure. Electroencephalogr Clin Neurophysiol 32: 281-294, 1972.

19. Veliskova J: Behavioral characterization of seizures in rats. In: Models of Seizures and Epilepsy. Elsevier Academic Press, Burlington, pp601-611, 2006.

20. Livak KJ and Schmittgen TD: Analysis of relative gene expression data using real-time quantitative PCR and the 2(-Delta Delta C(T)) method. Methods 25: 402-408, 2001.

21. Bagdy G, Kecskemeti V, Riba P and Jakus R: Serotonin and epilepsy. J Neurochem 100: 857-873, 2007.

22. Theodore WH: Does serotonin play a role in epilepsy? Epilepsy Curr 3: 173-177, 2003.

23. Gidal BE: Serotonin and epilepsy: The story continues. Epilepsy Curr 13: 289-290, 2013.

24. Airaksinen EM: Uptake of taurine, GABA, 5-HT, and dopamine by blood platelets in progressive myoclonus epilepsy. Epilepsia 20: 503-510, 1979.

25. Bateman LM, Li CS, Lin TC and Seyal M: Serotonin reuptake inhibitors are associated with reduced severity of ictal hypoxemia in medically refractory partial epilepsy. Epilepsia 51: 2211-2214, 2010.

26. Trindade-Filho EM, de Castro-Neto EF, de A Carvalho R, Lima E, Scorza FA, Amado D, Naffah-Mazzacoratti Mda G and Cavalheiro EA: Serotonin depletion effects on the pilocarpine model of epilepsy. Epilepsy Res 82: 194-199, 2008.

27. da Fonseca NC, Joaquim HP, Talib LL, de Vincentiis S, Gattaz WF and Valente KD: Hippocampal serotonin depletion is related to the presence of generalized tonic-clonic seizures, but not to psychiatric disorders in patients with temporal lobe epilepsy. Epilepsy Res 111: 18-25, 2015.

28. Cardamone L, Salzberg MR, Koe AS, Ozturk E, O'Brien TJ and Jones NC: Chronic antidepressant treatment accelerates kindling epileptogenesis in rats. Neurobiol Dis 63: 194-200, 2014. 
29. Esmail EH, Labib DM and Rabie WA: Association of serotonin transporter gene (5HTT) polymorphism and juvenile myoclonic epilepsy: A case-control study. Acta Neurol Belg 115: 247-251, 2015.

30. Yang K, Su J, Hu Z, Lang R, Sun X, Li X, Wang D, Wei M and Yin J: Serotonin transporter (5-HTT) gene polymorphisms and susceptibility to epilepsy: A meta-analysis and meta-regression. Genet Test Mol Biomarkers 17: 890-897, 2013.

31. Martinez A, Finegersh A, Cannon DM, Dustin I, Nugent A, Herscovitch $\mathrm{P}$ and Theodore WH: The 5-HT1A receptor and 5-HT transporter in temporal lobe epilepsy. Neurology 80: 1465-1471, 2013

32. Schenkel LC, Bragatti JA, Torres CM, Martin KC, Gus-Manfro G, Leistner-Segal S and Bianchin MM: Serotonin transporter gene (5HTT) polymorphisms and temporal lobe epilepsy. Epilepsy Res 95: 152-157, 2011.

33. Kobow $\mathrm{K}$ and Blümcke I: The methylation hypothesis: Do epigenetic chromatin modifications play a role in epileptogenesis? Epilepsia 52 (Suppl 4): S15-S19, 2011.

34. Serikawa T, Mashimo T, Kuramoro T, Voigt B, Ohno Y and Sasa M: Advances on genetic rat models of epilepsy. Exp Anim 64: 1-7, 2015.

35. Ran X, Li J, Shao Q, Chen H, Lin Z, Sun ZS and Wu J: EpilepsyGene: A genetic resource for genes and mutations related to epilepsy. Nucleic Acids Res 43 (Database issue): D893-D899, 2015.

36. Weber YG, Nies AT, Schwab M and Lerche H: Genetic biomarkers in epilepsy. Neurotherapeutics 11: 324-333, 2014.

37. Moon J, Lee ST, Choi J, Jung KH, Yang H, Khalid A, Kim JM, Park KI, Shin JW, Ban JJ, et al: Unique behavioral characteristics and microRNA signatures in a drug resistant epilepsy model. PloS one 9: e85617, 2014.

38. Jimenez-Mateos EM and Henshall DC: Epilepsy and microRNA. Neuroscience 238: 218-229, 2013.

39. Huang S, Zou X, Zhu JN, Fu YH, Lin QX, Liang YY, Deng CY, Kuang SJ, Zhang MZ, Liao YL, et al: Attenuation of microRNA-16 derepresses the cyclins D1, D2 and E1 to provoke cardiomyocyte hypertrophy. J Cell Mol Med 19: 608-619, 2015.

40. Li W, Qi Z, WeiZ, Liu S, Wang P, Chen Y and Zhao Y: Paeoniflorin inhibits proliferation and induces apoptosis of human glioma cells via microRNA-16 upregulation and matrix metalloproteinase-9 downregulation. Mol Med Rep 12: 2735-2740, 2015.
41. Mobarra N, Shafiee A, Rad SM, Tasharrofi N, Soufi-Zomorod M, Hafizi M, Movahed M, Kouhkan F and Soleimani M: Overexpression of microRNA-16 declines cellular growth, proliferation and induces apoptosis in human breast cancer cells. In Vitro Cell Dev Biol Anim 51: 604-611, 2015.

42. Bai M, Zhu X, Zhang Y, Zhang S, Zhang L, Xue L, Yi J, Yao S and Zhang X: Abnormal hippocampal BDNF and miR-16 expression is associated with depression-like behaviors induced by stress during early life. PLoS One 7: e46921, 2012.

43. Sun YX, Yang J, Wang PY, Li YJ, Xie SY and Sun RP: Cisplatin regulates $\mathrm{SH}-\mathrm{SY} 5 \mathrm{Y}$ cell growth through downregulation of BDNF via miR-16. Oncol Rep 30: 2343-2349, 2013.

44. Kilany A, Raouf ER, Gaber AA, Aloush TK, Aref HA, Anwar M, Henshall DC and Abdulghani MO: Elevated serum Bcl-2 in children with temporal lobe epilepsy. Seizure 21: 250-253, 2012.

45. Henshall DC, Clark RS, Adelson PD, Chen M, Watkins SC and Simon RP: Alterations in bcl-2 and caspase gene family protein expression in human temporal lobe epilepsy. Neurology 55: 250-257, 2000

46. Scharfman H: Does BDNF contribute to temporal lobe epilepsy? Epilepsy Curr 2: 92-94, 2002.

47. Kuramoto S, Yasuhara T, Agari T, Kondo A, Jing M, Kikuchi Y, Shinko A, Wakamori T, Kameda M, Wang F, et al: BDNF-secreting capsule exerts neuroprotective effects on epilepsy model of rats. Brain Res 1368: 281-289, 2011

48. Song MF, Dong JZ, Wang YW, He J, Ju X, Zhang L, Zhang YH, Shi JF and Lv YY: CSF miR-16 is decreased in major depression patients and its neutralization in rats induces depression-like behaviors via a serotonin transmitter system. J Affect Disord 178: 25-31, 2015

49. Zurawek D, Kusmider M, Faron-Gorecka A, Gruca P, Pabian P, Solich J, Kolasa M, Papp M and Dziedzicka-Wasylewska M: Reciprocal microrna expression in mesocortical circuit and its interplay with serotonin transporter define resilient rats in the chronic mild stress. Mol Neurobiol: Sep 22, 2016 (Epub ahead of print). 